\title{
Assessement of Candida utilis growth by voltammetric reduction of acids using microelectrodes
}

A. Rodrigues ${ }^{a}$, M.D. Geraldo ${ }^{a}$, M.F. Bento ${ }^{a,}$, F. Cássio $^{b}$

${ }^{a}$ Departamento de Química; ${ }^{b}$ Departamento de Biologia, Universidade do Minho, Campus de Gualtar, 4710-057 Braga, Portugal

*fbento@quimica.uminho.pt

\begin{abstract}
A voltammetric method for the evaluation of the microbial growth using platinum microelectrodes is proposed. This new method is based on the electrochemical reduction of acids produced by the yeast Candida utilis in liquid medium containing glucose as carbon and energy source and ammonium ions as nitrogen source. Current intensities, from linear sweep and square wave voltammetry, were compared with the corresponding values of absorbance at $640 \mathrm{~nm}\left(A_{640 \mathrm{~nm}}\right)$ resulting from the light scattering of cell suspensions. The equivalence between turbidimetry and electrochemical measurements was checked in experiments performed in growing cultures and in cell suspensions prepared from the dilution of cell cultures. The growth curves defined by either current intensities $\left(I_{\mathrm{P}}\right.$ or $\left.I_{\mathrm{L}}\right)$ or $A_{640 \mathrm{~nm}}$ measurements were similar and displayed characteristic growth phases in a closed system. Exponential growth rates estimated both from turbidimetry and voltammetric techniques were the same.
\end{abstract}

Keywords: voltammetry, microelectrodes, growth monitoring, yeast

\section{Introduction}

Microbial biomass is one of the most important quality indices in clinical, environmental and bioindustrial fields. This parameter can be measured directly either by dry weight mass or plate count. As these procedures are tedious and time consuming, several alternative indirect techniques, based on physical, chemical or electrochemical properties of microorganism cultures have been developed [1]. Turbidimetry is doubtless the most popular technique for biomass determinations since cells are roughly of constant size and the increase in cell number results in a proportional increase in the light scattering. Although this method is accurate and rapid, measurements can be meaningless if suspensions are not properly stirred, or in the presence of bubbles or suspended material.

The access to on-line and real-time biomass is of critical importance in bioprocesses monitoring, in order to control and optimise the cultivation process. For this purpose, optical techniques such as near-infrared spectroscopy demonstrated to be 
more suitable than turbidimetry [2]. Sensors and probes of different nature are also being developed [3].

Electrochemical techniques are known to be rapid, accurate and inexpensive and electrodes can be designed in order to be selective and have high mechanical stability even under harsh environmental conditions. Indeed, electrochemical methods have been applied to microbial biomass evaluation after the work of Richards et al. [4]. The release of ionic metabolites to the growth medium was monitorized by conductivity or impedancimetry and the variation of these signals was related to cellular growth and biomass [1]. Reported potentiometric methods were based on the detection of either molecular hydrogen produced by bacteria [5] or lipoic acid, which was provided to microbial cultures [6]. The potentiometric $\mathrm{pH}$ sensor has also been used as a way of controlling the addition of acid or basic titrants to attain constant $\mathrm{pH}$. The amount of added titrants was used as a measure of the acids / bases generated from the metabolic activity of yeasts and provided an indirect measure of the biomass [7]. Direct $\mathrm{pH}$ measurements are not usually used to assess the concentration of acids or bases due to the complexity of the culture media composition and cellular metabolism. Besides the weak acids and bases used in the culture medium preparation, others can arise from cellular metabolism, resulting in a buffer system highly intricate and in such condition $\mathrm{pH}$ measurements do not track the concomitant variation of acid concentrations related to microbial growth.

Amperometric methods using both different potential techniques and electrode materials were described [1], reporting the direct oxidation of cells using either bare carbon electrodes $[8,9]$ or modified electrodes [10,11]. The electron transfer between cells and the electrodes was facilitated by the physical placement of microbial cells at the electrode proximity. This can be achieved by different procedures involving either concentration of the cell suspensions $[9,11]$ or adsorptive steps prior the measurement [12]. The use of redox mediators added to the cell culture was also described [13,14].

In this work, we report a new electroanalytical method for microbial growth evaluation, which is based on the voltammetric cathodic currents of platinum microelectrodes in the cell cultures. The electrode surface was not submitted to any modification and measurements were carried out in a common two-neck flask with two electrodes. Growth medium composition was selected in order to meet the requirements of the microorganisms and without addition of a mediator. Electrochemical measurements were performed directly in cell culture samples without making any preconcentration or dilution.

\section{Experimental}

\subsection{Microorganism and growth conditions}

Candida utilis IGC 3039 (CBS 890) was maintained on a glucose containing medium (2\%, $w / v)$, peptone $(1 \%, w / v)$, yeast extract $(0.5 \%, w / v)$ and agar $(2 \% \mathrm{w} / \mathrm{v})$. For growth in a liquid medium, a mineral medium with $\left(\mathrm{NH}_{4}\right)_{2} \mathrm{SO}_{4},(0.5 \%, w / v)$ as nitrogen source, $\mathrm{KH}_{2} \mathrm{PO}_{4} \quad(0.5 \%, \quad w / v)$, $\mathrm{MgSO}_{4} .7 \mathrm{H}_{2} \mathrm{O}(0.05 \%, w / v), \mathrm{CaCl}_{2} .2 \mathrm{H}_{2} \mathrm{O}(0.01 \%$, $w / v)$ supplemented with vitamins [15] and glucose $(2 \%, w / v)$, as carbon and energy source was used. Cells were grown in $500 \mathrm{~mL}$ Erlenmeyer flasks in a void volume of half of the total flask volume with mechanical shaking (120 r.p.m.) at $26^{\circ} \mathrm{C}$. 


\subsection{Measurement of growth and determination of cell numbers}

Growth was monitored through the increase of absorbance measured at $640 \mathrm{~nm}, A_{640 \mathrm{~nm}}$, (Spectronic 21, Bausch \& Lomb). The number of cells of $C$. utilis was determined through direct counting in a Neubauer chamber.

\subsection{Sample preparation for voltammetry}

Voltammetric assays were performed in $5 \mathrm{~mL}$ samples obtained from either cell growing cultures, diluted cell suspensions, medium-free of cells or cell resuspended in fresh-culture medium. Diluted cell suspensions $\left(0.194<A_{640 \mathrm{~nm}}<4.012\right)$ were prepared by dilution of growing cultures with freshculture medium. To obtain the culture medium-free of cells, the cells were harvested by centrifugation at $8000 \mathrm{~g}$. To prepare cell suspensions in freshculture medium, harvested cells were washed three times with the mineral medium prior suspension in the same volume of medium.

\subsection{Equipment}

Measurements were performed using a potentiostat (Autolab type PGSTAT30, Ecochemie) controlled by the GPES 4.6 software, provided by Ecochemie. Steady state voltammograms from linear sweep voltammetry (LSV) were obtained using $50 \mathrm{mV} \mathrm{s}^{-1}$ scan rate. The optimised parameters for square wave voltammetry (SWV) were $f=8 \mathrm{~Hz}, \Delta E=5 \mathrm{mV}$ and $E_{\mathrm{SW}}=25 \mathrm{mV}$.

All voltammetric experiments were carried out, at room temperature, in a two-electrode arrangement placed in a common two-neck flask into a Faraday cage. The reference / counter electrode was a saturated calomel electrode (SCE). All potentials are quoted versus SCE. The working electrodes were platinum microdisks of 25.0 and $12.5 \mu \mathrm{m}$ radius, made of cross section of metal wires (Goodfellows) sealed into soft glass. The electrodes were polished by successive steps, first using fine abrasive papers and then with alumina $(0.3 \mu \mathrm{m}$ size, Tonerde, ATM) on a wet tissue (Mambo, Ziesmer). The electrodes were washed with water and dried before use. Prior to each experiment the electrode surface was polished with $0.05 \mu \mathrm{m}$ alumina, rinsed with water, polished with a wet polishing cloth, rinsed again and dried. Electrode diameters were calibrated by measuring the limiting voltammetric current for a known concentration of ferricyanide, using the following diffusion coefficient: $7.2 \times 10^{-6}$ $\mathrm{cm}^{2} \mathrm{~s}^{-1}$ [16]. All current intensity data correspond to the average of at least five determinations. In plots the error bars are not visible due to the high reproducibility of data.

\section{Results and Discussion}

3.1. Characterization of the culture medium and cell suspensions by linear sweep and square wave voltammetry.

The square wave voltammogram b) in Fig. 1, obtained in a suspension of cells of $C$. utilis grown in a mineral medium with glucose, displayed an important reduction process at approximately -0.47 $\mathrm{V}$ versus SCE. Linear sweep voltammograms recorded at slow scan rates $\left(50 \mathrm{mV} \mathrm{s}^{-1}\right)$ presented a single waveform signal characteristic of steady state voltammetry, close to $-0.45 \mathrm{~V}$ versus SCE (not shown). Recorded signals from both techniques are well defined and were associated with the presence of the cells, as they were not observed in the culture medium prior cell inoculation (curve a) Fig. 1 for SWV). The major constituents of the culture medium are minerals, $\left(\mathrm{NH}_{4}\right)_{2} \mathrm{SO}_{4}, \mathrm{MgSO}_{4}, \mathrm{CaCl}_{2}$, and a buffer system based on $\mathrm{KH}_{2} \mathrm{PO}_{4}, \mathrm{pH}=4.1 \pm 0.1$. 


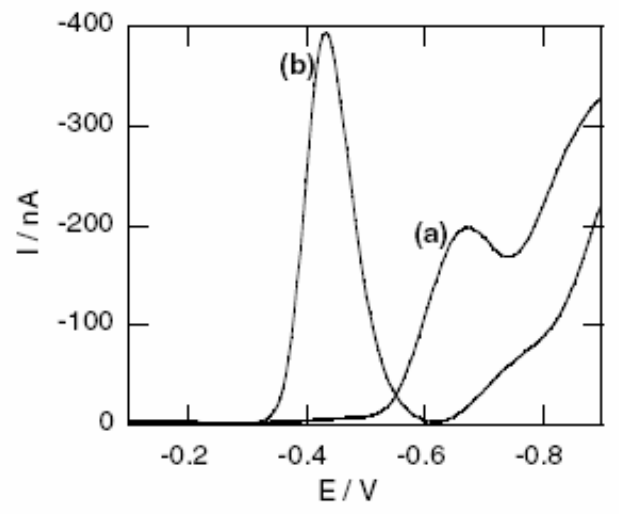

Fig. 1. Square wave voltammograms recorded with a $25.0 \mu \mathrm{m}$ radius platinum microelectrode in: (a) mineral medium with glucose and (b) suspension of cells of C. utilis $\left(\mathrm{A}_{640 \mathrm{~nm}}=4.012\right)$ grown in the same medium.

The voltammetric characterization of this solution was carried out by sequential addition of each constituent and the peak occurring at $-0.70 \mathrm{~V}$ was attributed mainly to the reduction of the dihydrogenphosphate anion. To identify the species involved in the electrochemical process voltammetric experiments were performed in i) a culture medium-free of cells and ii) cells resuspended in fresh culture medium. In the first case, voltammograms were similar to those obtained in cell suspensions from growing cultures (curve b) Fig. 1), while voltammograms displayed in the second case were identical to those of the culture medium (curve a) Fig. 1), where the electrochemical signal at $-0.45 \mathrm{~V}$ was not detected. Therefore, the voltammetric process can be related to chemical species in solution produced by the cells during their growth and not to the physical presence of the cells.

\subsection{Effect of culture dilution on the voltammetric} response

A cell suspension obtained from growing cells of $C$. utilis was diluted with the culture medium and the resulting cell suspensions were analysed by linear sweep voltammetry (LSV) and square wave voltammetry (SWV). The degree of dilution of each suspension was expressed by the dilution factor, $F_{\mathrm{d}}$ $\left(F_{\mathrm{d}}=v_{\text {original }} / v_{\text {total }}\right)$. Voltammograms obtained from the original cell suspension by LSV (curve a) Fig. 2-A) and SWV (curve a) Fig. 2-B) exhibited a unique process. A decrease in the voltammetric current intensity was observed as the concentration of cells in suspension diminished. Voltammograms obtained in the most diluted cell suspensions displayed a second process at more negative potentials, which is characteristic of the culture medium used to perform the dilutions.

The shape and position of voltammetric peak and wave changed slightly with the concentration of cells. As cell suspensions were diluted
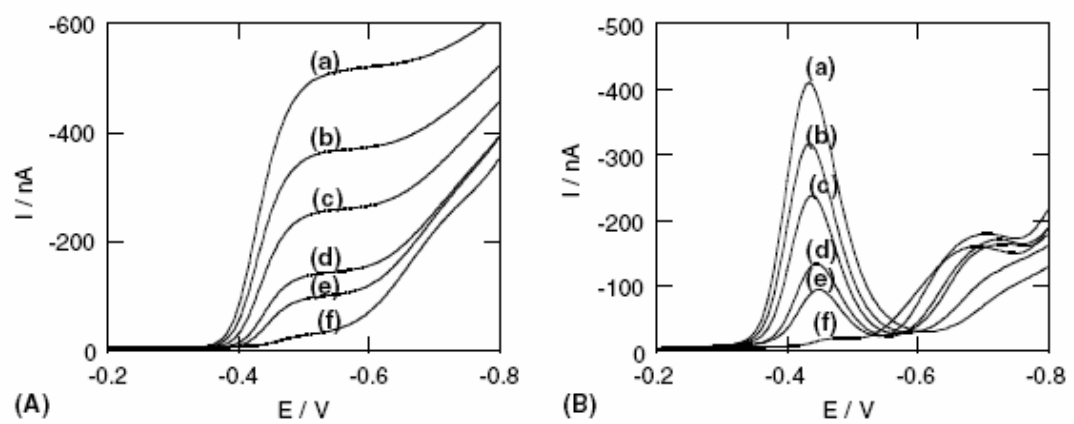

Fig. 2. Voltammetric responses of a $12.5 \mu \mathrm{m}$ radius platinum microelectrode from LSV (A) and SWV (B) in cell suspensions obtained from dilution of a $C$. utilis culture $\left(\mathrm{A}_{640 \mathrm{~nm}}=4: 012\right)$ with the culture medium. Analysed suspensions are assigned with the corresponding $\mathrm{F}_{\mathrm{d}}$ values: (a) 1 , (b) 0.700 , (c) 0.500 , (d) 0.300 , (e) 0.200 and (f) 0.0500 . 
voltammetric signals were deviated towards more negative potentials (approximately $14 \mathrm{mV}$ for $E_{\mathrm{P}}$ and $20 \mathrm{mV}$ for $E_{1 / 2}$ ). Simultaneously, peak tended to be tighter in relation to its height and the wave $\left|E_{3 / 4}-E_{1 / 4}\right|$ value decreased about $10 \mathrm{mV}$.

The direct proportionalities obtained for current intensities, $I_{\mathrm{P}}(\mathrm{SWV})$ and $I_{\mathrm{L}}(\mathrm{LSV})$, against the dilution factor (Fig. 3-A), demonstrated that the concentration of chemical species increased proportionally with the dilution factor and simultaneously the electrochemical process is diffusion controlled. In spite of the great variation in the concentration of cells, which might affect the medium physical properties, like viscosity, the diffusion coefficient of the electroactive species did not seem to be significantly influenced. Absorbance followed also a direct proportionality with $F_{\mathrm{d}}$, which regression equation is $A_{640 \mathrm{~nm}}=-0.1 \pm 0.1+$
(4.4 \pm 0.3$) \quad F_{\mathrm{d}} ; \quad(\mathrm{r}=0.997, \mathrm{n}=11)$, since data are acquired in diluted solutions $\left(A_{640 \mathrm{~mm}}<0.8\right)$. As a consequence of both direct proportionalities of $I_{\mathrm{P}}$ and $I_{\mathrm{L}}$ versus $F_{\mathrm{d}}$ and $A_{640 \mathrm{~nm}}$ versus $F_{\mathrm{d}}$, a direct proportionality was also observed between current intensities $\left(I_{\mathrm{P}}\right.$ and $\left.I_{\mathrm{L}}\right)$ and absorbance (Fig. 3-B), establishing the equivalence of the represented parameters. Detection limits of the voltammetric methods, estimated from statistical analysis of the regression lines in Fig. 3-A, were expressed as dilution factors and then converted to the corresponding absorbance values (through the correlation $A_{640 \mathrm{~nm}}$ versus $F_{\mathrm{d}}$ ). The value of 0.14 $\left(A_{640 \mathrm{~nm}}\right)$ was estimated as the detection limit for LSV and corresponded to $10^{7}$ cells $\mathrm{mL}^{-1}$, whereas for SWV the value was $0.09\left(A_{640 \mathrm{~nm}}\right)$ and corresponded to $8 \times 10^{6}$ cells $\mathrm{mL}^{-1}$.
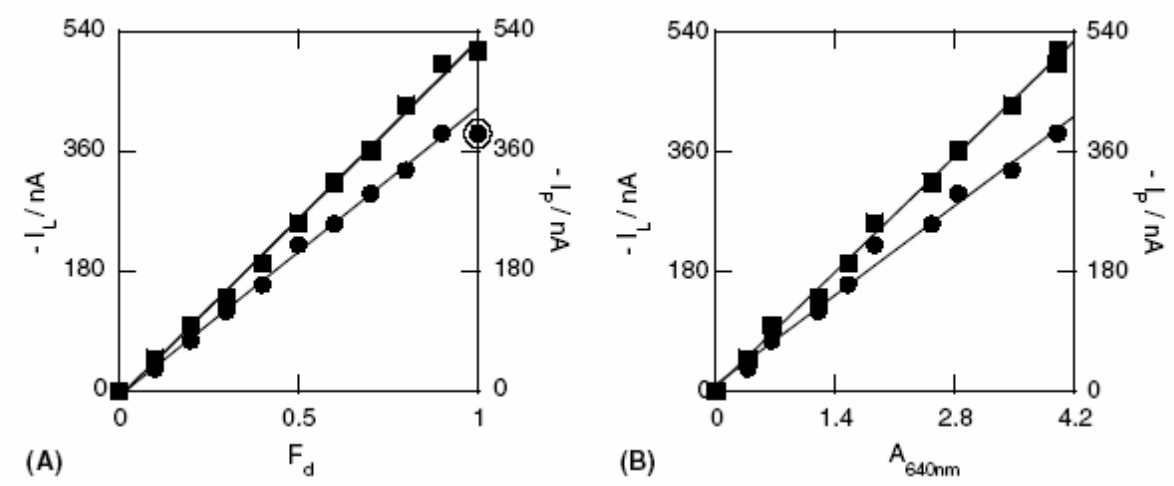

Fig. 3. Representation of $\mathrm{I}_{\mathrm{P}}(\bullet)$ and $\mathrm{I}_{\mathrm{L}}(\bullet)$ versus either $\mathrm{F}_{\mathrm{d}}(\mathrm{A})$ or $\mathrm{A}_{640 \mathrm{~nm}}(\mathrm{~B})$. Experimental conditions were the same as in Fig. 2. The assigned point in plot $\mathrm{A}$ was not used for the definition of the regression line and was not considered in the plot $\mathrm{B}$
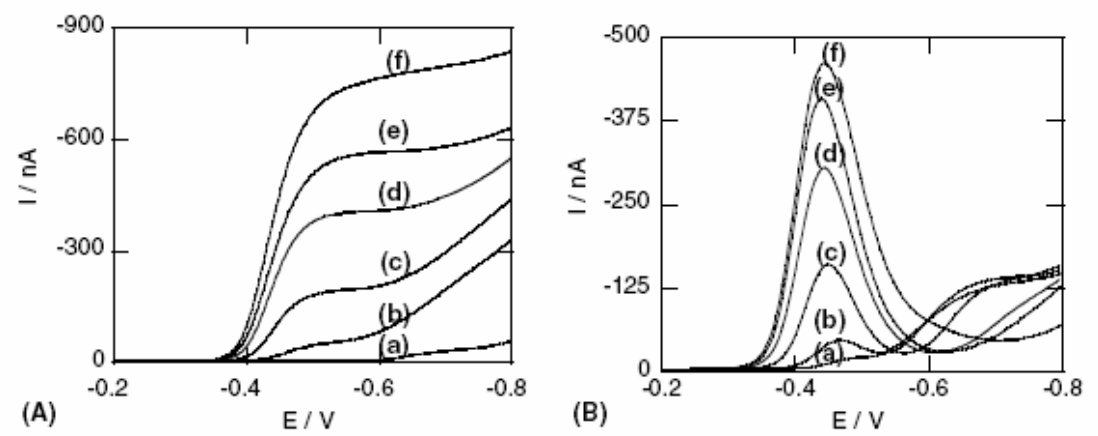

Fig. 4. Voltammograms from LSV (A) and SWV (B) obtained in a culture of C. utilis. Time of growth: (a) 0, (b) 2.25, (c) 5.50, (d) 7.77, (e) 8.83 and (f) $12.5 \mathrm{~h}$. Assays were performed with a $25.0 \mu \mathrm{m}$ radius platinum microelectrode. 

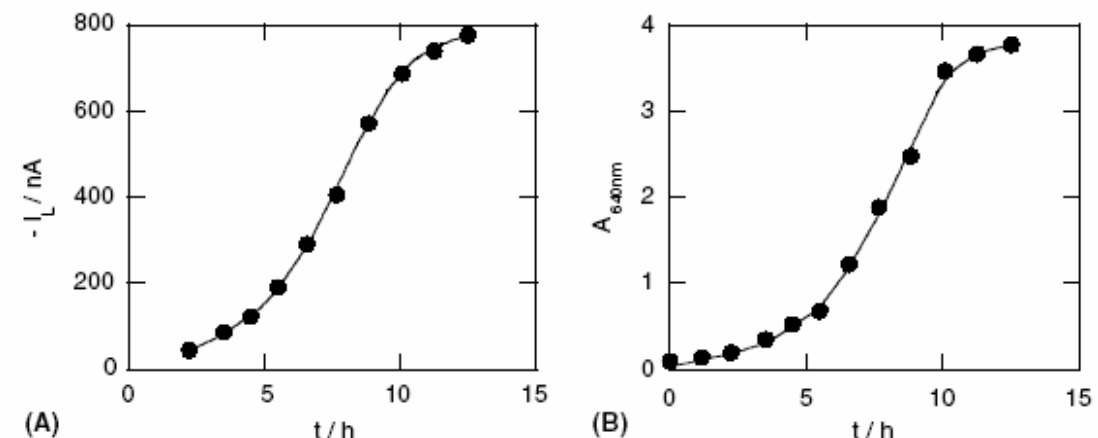

Fig. 5. Growth curves of C. utilis obtained from optical (A) and LSV measurements (B). The experimental conditions were the same as in Fig. 4.

\subsection{Monitoring the growth of Candida utilis}

The growth of the yeast $C$. utilis was followed by voltammetric and optical measurements. Voltammograms acquired along time by LSV and SWV are presented in Fig. 4-A and 4-B, respectively. At earliest time, voltammograms recorded in the cell culture (curves a) in Fig. 4-A and 4-B) exhibit the characteristic feature of those from culture medium prior cell inoculation (curve a) in Fig. 1 for SWV). Voltammograms acquired thereafter showed that the current intensity at -0.45 $\mathrm{V}$ increased whereas the current intensity at $-0.7 \mathrm{~V}$ (characteristic of the culture medium) decreased. In addition, the voltammetric wave position was shifted by $20 \mathrm{mV}$ towards positive potentials and the corresponding $\left|E_{3 / 4}-E_{1 / 4}\right|$ value changed from 40 to $60 \mathrm{mV}$. The voltammetric peak tended to be wider in relation to its height and shifted about the same magnitude as the wave, in accordance with the results obtained from diluted cell suspensions. Electrochemical and optical data acquired during cell growth against time are presented in Fig. 5-A and 5-B, respectively. In both plots characteristic phases of the cellular growth curve in a closed system are recognized, nevertheless small differences can be noticed on earlier times of growth. The absence of the two first points in Fig 5-

A, in comparison to Fig 5-B, reflects differences in the detection limits of voltammetric and turbidimetric methods.

To check the equivalence between electrochemical and optical measurements obtained in growing cultures, absorbance and current intensity data were normalized to arbitrary reference values (acquired at $t=4.50 \mathrm{~h}$ ). In Fig. 6 values of the normalized variables are represented as $I_{\mathrm{L}, \mathrm{N}}$ versus $A_{640 \mathrm{~nm}, \mathrm{~N}}$, as well as the straight line corresponding to $\mathrm{Y}=\mathrm{X}$. The fulfilment of this correlation demonstrates that both measurements have a similar variation along time. Indeed, all the experimental data fitted this correlation except the three last points where current intensities seemed to increase less than the corresponding absorbance values.

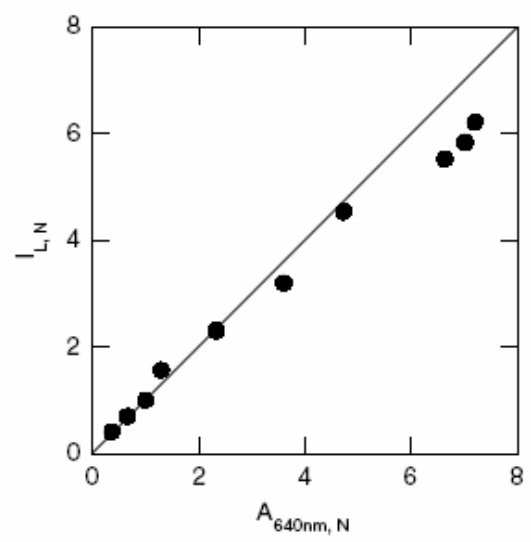

Fig. 6. Plot of normalized limiting current $(\mathrm{IL} ; \mathrm{N})$ versus normalized absorbance (A640 nm;N) calculated from data of Fig. 5. The coordinated pair used for normalization was $(\mathrm{A}=0.523 ; \mathrm{IL}=$ $-122.18 \mathrm{nA}$ ). The line corresponds to $\mathrm{Y}=\mathrm{X}$. 

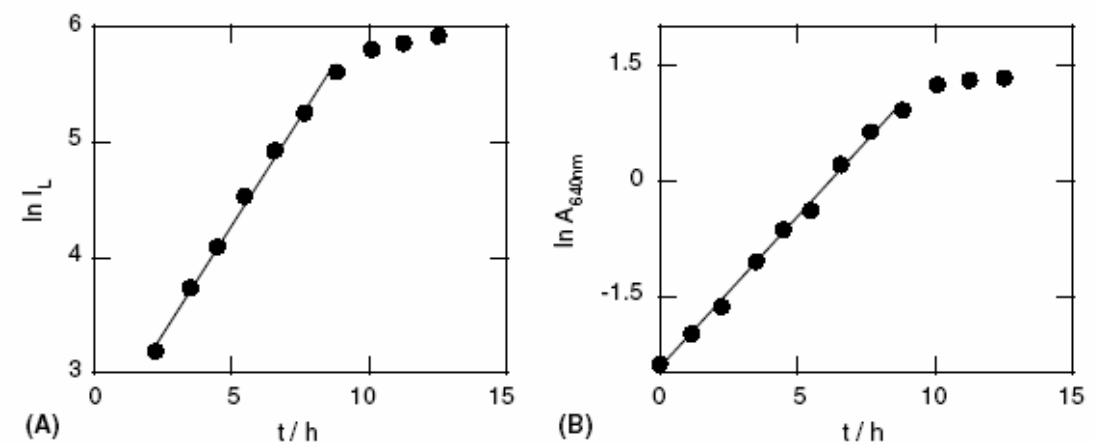

Fig. 7. Semi-logarithmic plot of limiting current (A) and absorbance (B) versus time obtained from a growing culture of C. utilis. Lines were obtained from linear regression, using data from the exponential growth phase.

Table 1. Specific growth rates $\left(\mu_{\max }\right)$ of $C$. utilis estimated from absorbance $\left(A_{640 \mathrm{~nm}}\right)$ and voltammetric (LSV and SWV) data, using 12.5 and $25.0 \mu \mathrm{m}$ radius microelectrode.

\begin{tabular}{|c|c|c|c|}
\hline Technique & Microelectrode radius / $\mu \mathrm{m}$ & $\mu_{\max } / \mathrm{h}^{-1}$ & Correlation coefficient of the semi-logaritmic plot \\
\hline Turbidimetry $^{\mathrm{a}}$ & & $0.39 \pm 0.02$ & 0.998 \\
\hline \multirow[t]{2}{*}{$\mathrm{LSV}^{\mathrm{a}}$} & 25.0 & $0.39 \pm 0.05$ & 0.995 \\
\hline & 12.5 & $0.37 \pm 0.03$ & 0.998 \\
\hline \multicolumn{2}{|l|}{ Turbidimetry $^{\mathrm{b}}$} & $0.36 \pm 0.02$ & 0.997 \\
\hline \multirow[t]{2}{*}{$S W V^{b}$} & 25.0 & $0.37 \pm 0.05$ & 0.98 \\
\hline & 12.5 & $0.36 \pm 0.04$ & 0.99 \\
\hline
\end{tabular}

The exponential phase of microbial growth represents a period of logarithmic cell doublings at constant and maximum specific growth rate $\left(\mu_{\max }\right)$ according to $x=x_{0} \mathrm{e}^{\mu \max t}$, where $x_{0}$ and $x$ are the cell mass in time 0 and $t$, respectively [17]. Thus, a plot of $\ln x$ versus $t$ is linear with the slope being $\mu_{\max }$. Fig. 7 shows the plots of the logarithm of either $A_{640 \mathrm{~nm}}$ or $I_{\mathrm{L}}$ versus $t$ (data from Fig. 5) from which similar values for $\mu_{\max }$ were estimated (Table 1). In addition, the linear dependence of both variables with time until 9 hours of growth showed similar portraits of the exponential growth phase assessed by voltammetric and turbidimetric methods.

Identical studies were carried out by LSV with a $12.5 \mu \mathrm{m}$ radius microelectrode and by SWV with either 12.5 or $25.0 \mu \mathrm{m}$ radius microelectrode. The estimated values of specific growth rates of $C$. utilis were similar in all experimental conditions (Table 1).

\subsection{Identification of electroactive species released by cells during growth}

Considering the potential values of $E_{1 / 2}$ or $E_{\mathrm{P}}$ and the acidification of the medium during the growth of $C$. utilis it can be inferred that the electroactive species are acids generated by the metabolic activity of the cells, as a similar range of potential was reported for the electrochemical reduction of acids with platinum microelectrodes $[18,19]$.

Voltammetric responses of hydrogen ion as well as carboxylic acids can occur at very close potentials, depending on the strength of the weak acid $[18,19]$. 
Reduction of protons from the dissociation of weak acids occurs via a CE mechanism with a fast chemical reaction for acids with dissociation constants larger than $1 \times 10^{-6}$, displaying a limiting current controlled by the diffusion of the acid $[19,20]$.

Extracellular medium acidification in cell cultures of microorganisms has been associated with growth processes and the cell metabolic activity was recognized as its main origin (see references in [7]). Several processes can contribute to this acidification, such as production of $\mathrm{CO}_{2}$ and carboxylic acids and the assimilation of nitrogen. Studies in growing cultures of C. utilis [7], Saccharomyces cerevisiae [22] and Escherichia coli [21,23] demonstrated that the nitrogen assimilation pathway is the most important process for the overall acidification, when ammonium ions are used as nitrogen source [7,21,22]. In batch and chemostat cultures of microorganisms, biomass formation rates were assessed by means of the amount of added titrant to a constant value of $\mathrm{pH}$ [7,21-23]. This method relies upon the accuracy of $\mathrm{pH}$ data obtained from glass electrodes immersed in the cell culture. As the $\mathrm{pH}$ is a measure of hydrogen ion activity its mean in terms of proton concentration depends on the composition similarity between culture media and standard solutions used for calibration. Besides, the variation of medium composition during cell growth can affect either ion strength or liquid junction potentials that are incorporated in potentiometric data, making $\mathrm{pH}$ measurements meaningless. Furthermore, charged colloidal suspensions are likely to occur as yeast cells have micrometric dimensions which turns unfeasible the use of standard $\mathrm{pH}$ glass electrodes.

\section{Conclusions}

The monitoring of current intensity, resulting of the reduction of acids generated by the metabolic activity of cells of $C$. utilis, grown in a medium with glucose as carbon and energy source and ammonium ions as nitrogen source, was used as an alternative approach to follow microbial growth. Estimated growth rates from either current intensities (SWV and LSV) or absorbance were identical. The high linearity limit of the electrochemical method enabled the performance of direct analysis in suspensions with higher concentration of cells than the optical method, avoiding thus dilution steps. Therefore the proposed electroanalytical method is suitable to perform online measurements in a flow system or directly in cell cultures.

As the electroanalytical signal is due to chemical species in solution, it is not influenced by the cellular morphology. This is in opposition to turbidimetry, which cannot be used when microorganisms exhibit mycelial growth or cell aggregates. This property makes the voltammetric method very attractive once dimorphic transitions between unicellular yeast cells to filamentous forms (and vice versa) are frequently found in various industrially important yeasts [24]. Furthermore, the proposed method can be especially advantageous as voltammetric measurements are immune to the samples turbidity associated with the presence of suspended material or gas bubbles, which can happen during biotechnological processes.

Research is currently being conducted in order to extend this method to a wider range of experimental conditions. Experiments with either different microorganisms or carbon sources will be performed since different microbial metabolic species can be produced, which might interfere with the electrochemical signal. 


\section{Acknowledgements}

This work was supported by POCTI/QUI/39525/2001.

\section{References}

[1] N.S. Hobson, I. Tothill, A.P.F. Turner, Biosens. Bioelectron. 5 (1996) 455.

[2] Z. Ge, A.G. Cavinato, J.B. Callis, Anal. Chem. 66 (1994) 1354.

[3] P. Harms, Y. Kostov, G. Rao, Curr. Opin. Biotech. 13 (2002) 124.

[4] J.C. Richards, A.C. Jason, G. Hobbs, D.M. Gibson, R.H. Christie, J. Phys. E. Sci. Instrum. 11 (1978) 560.

[5] J.R. Wilkins, G.E. Stoner, E.H. Boykin, Appl. Environ. Microbiol. 27 (1974) 949.

[6] G-A. Junter, Bioelectroch. Bioener. 12 (1984) 81.

[7] J.I. Castrillo, I. de Miguel, U.O. Ugalde, Yeast 11 (1995) 1353.

[8] T. Matsunaga, Y. Namba, Anal. Chem. 56 (1984) 798.

[9] Y.X. Ci, J. Feng, Z.W. Jiang, D.Z. Luo, Bioelectroch. Bioener. 43 (1997) 293.

[10] T. Matsunaga, T. Nakajima, Appl. Environ. Microb. 50 (1985) 238.

[11] S. Han, X. Li, G. Guo, Y. Sun, Z. Yuan, Anal. Chim. Acta 405 (2000) 115.
[12] M. Okochi, T. Matsunaga, Electrochim. Acta 42 (1997) 3247.

[13] A.P.F. Turner, G. Ramsay, I.J. Higgins, Biochem. Soc. T. 11 (1983) 455.

[14] P. Ertl, B. Unterladstaetter, K. Bayer, S.R. Mikkelsen, Anal. Chem. 72 (2000) 4949.

[15] N. Vanuden, Arch. Microbiol. 58 (1967) 155.

[16] K.T. Kawagoe, J.A. Jankowski, R.M. Wightman, Anal. Chem. 63 (1991)1589.

[17] M.T. Madigan, J.M. Martinko, J. Parker, Brock Biology of Microorganisms, Prentice Hall, $9^{\text {th }}$ edition, New Jersey, 2000, chap. 5.

[18] M. Ciskowska, Z. Stojek, S.E. Morris, J.G. Osteryoung, Anal. Chem. 64 (1992) 2372.

[19] S. Daniele, I. Lavagnini, M.A. Baldo, F. Magno, J. Electroanal. Chem. 404 (1996) 105.

[20] M. Fleischmann, F. Lasserre, J. Robinson, D. Swan, J. Electroanal. Chem. 177 (1984) 97.

[21] N.T. Eriksen, I. Kratchmarova, S. Neve, K. Kristiansen, J.J.L. Iversen, Biotechnol. Bioeng. 75 (2001) 355 .

[22] A. Vicente, J.I. Castrillo, J.A. Teixeira, U. Ugalde, Biotechnol. Bioeng. 58 (1998) 445.

[23] M.L. Christensen, N.T. Eriksen, Enzyme Microb. Tech. 31 (2002) 566.

[24] G.M. Walker, Yeast Physiology and Biotechnology, John Wiley \& Sons, New York, 1998, chap. 6. 